\title{
Nonstructural Protein 1 of Tick-Borne Encephalitis Virus Induces Oxidative Stress and Activates Antioxidant Defense by the Nrf2/ARE Pathway
}

\author{
Yulia V. Kuzmenko Olga A. Smirnova Alexander V. Ivanov \\ Elizaveta S. Starodubova Vadim L. Karpov \\ Engelhardt Institute of Molecular Biology, Russian Academy of Sciences, Moscow, Russia
}

\section{Keywords}

Nonstructural protein 1 - Tick-borne encephalitis - Oxidative stress - Reactive oxygen species · Antioxidant defense

\section{Abstract}

Background: Infection with tick-borne encephalitis virus (TBEV) causes pathological changes in the central nervous system. However, the possible redox alterations in the infected cells that can contribute to the virus pathogenicity remain unknown. Objective: In the current study we explored the ability of TBEV nonstructural protein 1 (NS1) to induce oxidative stress and activate antioxidant defense via the nuclear factor (erythroid-derived-2)-like 2/antioxidant response element (Nrf2/ARE) pathway. Methods: HEK 293T cells were transfected with plasmid encoding NS1 protein, and the production of reactive oxygen species (ROS) was measured using oxidation-sensitive dyes, the activation of the ARE promoter was estimated using a reporter plasmid, and the expression of phase II detoxifying enzymes was quantified by measuring their mRNA levels using RT-qPCR. Results: A high level of ROS production was detected in cells transfected with NS1-expressing plasmid. In addition, this protein activated the promoter with an ARE and upregulated the transcription of ARE-dependent genes that encode phase II enzymes. Conclusion: TBEV NS1 protein both triggers ROS production and activates a defense Nrf2/ARE pathway. These data suggest that a role of redox-mediated processes in TBEV-induced damage of the central nervous system should also be explored. These data can contribute to a better understanding of TBEV pathogenicity, further improvement of TBE treatment, and the development of vaccine candidates against this infection. @ $2016 \mathrm{~S}$. Karger AG, Basel

\section{Introduction}

Tick-borne encephalitis virus (TBEV) can cause severe infection of the central nervous system (CNS) in humans. This disease is characterized by a biphasic course. Symptoms of the first stage include acute febrile illness that is followed by an apparent recovery period. A spectrum of neurological manifestations ranging from headaches to meningitis, meningoencephalitis, and menongoencephalomyelitis is registered during the second stage [1]. TBEV

\section{KARGER}

E-Mail karger@karger.com

www.karger.com/int
C 2016 S. Karger AG, Base

$0300-5526 / 16 / 0592-0111 \$ 39.50 / 0$
Elizaveta S. Starodubova, $\mathrm{PhD}$

Engelhardt Institute of Molecular Biology, Russian Academy of Sciences Vavilova 32

RU-119991 Moscow (Russia)

E-Mail estarodubova@yandex.ru 
causes damage within the CNS. Neurons and other CNS cells are injured by the virus directly and indirectly due to induction of an immunopathogenic response [2]. However, the molecular mechanisms that trigger pathophysiological events during tick-borne encephalitis (TBE) are insufficiently explored.

Viral infections and the accompanied production of interferons often promote the production of oxygen radicals in cells. Reactive oxygen species (ROS) can act as key elements in antiviral defense [3], but they can also be harmful for the host cells and contribute to the pathogenesis of a disease. Oxidative stress has been observed during many acute and chronic viral infections such as influenza [4], dengue fever [5], HIV [6], and hepatitis B and C $[7,8]$. In TBE patients the presence of viral antigens in CNS lesions [9] and increased levels of proinflamatory cytokines [10] have been noted. These factors can promote the generation of oxygen-derived free radicals. Indeed, elevated levels of lipid peroxidation products have been observed in cerebrospinal fluid of TBE patients, indicating its relevance to the disease pathophysiology [11]. However, no direct data are available on induction of oxidative stress during infection with TBEV despite its importance for a better understanding of the mechanisms of virus pathogenesis.

TBEV belongs to the Flaviviridae family composed of small enveloped viruses with the positive-sense RNA genome that encodes a single polyprotein [12]. After processing of the polyprotein, 3 structural and 7 nonstructural proteins are generated. Amino acid substitutions in several proteins can affect the neuropathogenesis of TBEV [2], and one of these proteins is nonstructural protein 1 (NS1) [13]. Highly conserved NS1 plays an important role in flavivirus genome replication and immune evasion [14]. In the infected cell NS1 is localized on the membrane of the endoplasmic reticulum (ER) as a homodimer and it is involved in viral RNA replication. During the late stages of infection hexameric NS1 is secreted and interacts with complement components of the immune system [15]. Despite the high importance of NS1, there is not much information about its interaction with cell host components. There is only controversial data on the implication of NS1 in TLR3 signaling [16-18]. In the case of other viruses, the induction of oxidative stress is often associated with viral nonstructural proteins, such as HIV Tat [19], Vpr [20], and RT [21], HCV core and NS5A $[7,22,23]$ parvovirus H-1 NS1 [24], and the human parvovirus B19 NS1 protein [25]. In the current study we explore ability of TBEV NS1 to affect the redox state of the host cell.

\section{Materials and Methods}

\section{Plasmids}

A DNA fragment corresponding to 2,383-3,513 nucleotides of the TBE full genome (Neudoerfl strain, GenBank U27495.1) was generated synthetically by assembling the oligonucleotides and cloned into the BamHI/EcoRI site of the $\mathrm{pVax}$ vector (Invitrogen). All of these procedures were performed by the company Evrogen, Russia. The plasmids pVax-core and pcDNA3.1-NS5B encoding HCV core and HCV NS5B, respectively, have been described previously $[22,26]$.

\section{Cell Lines and Transfection}

HEK 293T and HeLa cells were cultured in Dulbecco's modified Eagle's medium (DMEM) supplemented with 10\% fetal bovine serum (HyClone), $100 \mu \mathrm{g} / \mathrm{mL}$ penicillin, and $100 \mu \mathrm{g} / \mathrm{mL}$ streptomycin. All cell lines were incubated at $37^{\circ} \mathrm{C}$ with $5 \% \mathrm{CO}_{2}$. The day before transfection the cells were seeded, and transfection was performed using Lipofectamine LTX (Invitrogen) following the manufacturer's instructions.

\section{Western Blot}

Two days after transfection the cells were collected and lysed in SDS-loading buffer. Western blot was performed as previously described [27]. Blots were stained with monoclonal anti-NS1 clone 4C4 (BioSan) and HRP-conjugated anti-mouse IgG antibody (Jackson). Then the blots were stripped and stained with monoclonal anti-actin clone AC-15 (Sigma) antibodies.

\section{Confocal Immunofluorescence Microscopy}

HeLa cells were seeded onto coverslips in 6-well plates and transfected the next day. Two days after transfection the cells were fixed with $2 \%$ paraformaldehyde for $15 \mathrm{~min}$ and permeabilized with $0.1 \%$ Triton X-100 at room temperature. Then the samples were incubated in blocking solution (5\% BSA in PBS) for $30 \mathrm{~min}$, followed by staining with a mixture of monoclonal anti-NS1 antibody 4C4 (1:100; BioSan) and rabbit polyclonal anti-calreticulin (1:100; Abcam) as the first antibodies and an FITC-labeled antimouse IgG antibody (1:50; Jackson) and TRITC-labeled antimouse IgG antibody (1:50; Sigma) as the secondary antibodies. The coverslips were mounted with Vectashield containing DAPI (Vector Laboratories), and the cells were visualized using a Leica TCS SP5 confocal microscope.

\section{ROS Measurement}

The production of intracellular ROS was measured by epifluorescence of reporter dyes as described previously [21, 22, 28]. Two days after transfection the growth medium was removed and cells were incubated in DMEM containing $25 \mu \mathrm{M}$ dichlorofluorescein diacetate $\left(\mathrm{DCFH}_{2} \mathrm{DA}\right)$ or $25 \mu \mathrm{M}$ dihydroethidium (DHE) for 30 min. The fluorescence intensities were measured in PBS using a Chameleon V plate reader (Hidex Ltd.) with excitation/emission lengths for $\mathrm{DCFH}_{2} \mathrm{DA} 485 / 535 \mathrm{~nm}$ and DHE 510/590 nm. Positive control cells were transfected with pVax-core encoding the HCV core protein that triggers ROS production, or with the $\mathrm{pVax}$ vector with subsequent treatment with $100 \mu \mathrm{M}$ tert-butylhydroquinone (tBHQ) or $100 \mu \mathrm{M} \mathrm{H}_{2} \mathrm{O}_{2}$ for $1 \mathrm{~h}$ before incubation with the dyes. As a negative control, the cells were transfected either with the plasmid encoding the HCV NS5B protein or with the empty pVax vector. 


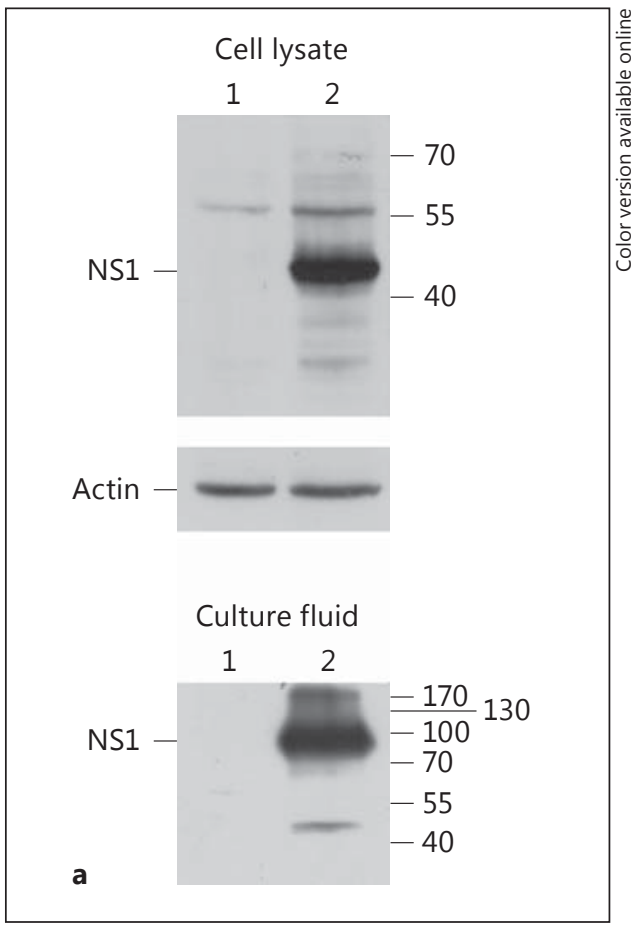

Fig. 1. Expression of the nonstructural protein 1 (NS1) protein in cells. a Western blot with anti-NS1 (monoclonal 4C4; BioSan) and anti-actin (monoclonal AC-15; Sigma) antibodies of lysate HEK 293T-transfected cells. b Localization of the NS1 protein in transfected HeLa cells. Cells were stained with anti-NS1 (field 1) and anti-calreticulin (field 2) antibodies. Overlay of signals (field 3).

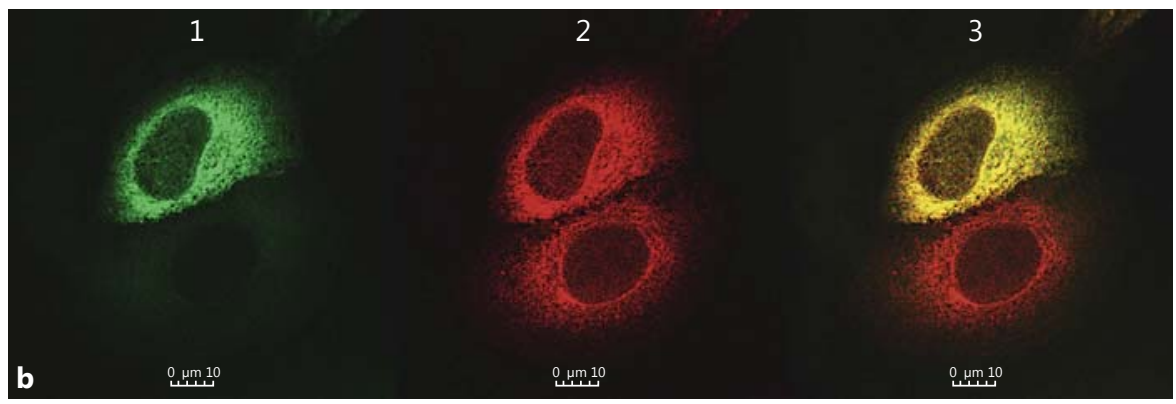

Activation of the Antioxidant Response Element Promoter

A reporter assay was used for estimation of antioxidant response element (ARE) upregulation as previously described [22]. HEK 293T cells were transfected by a mixture of pVax-derived plasmid and pP-ARE plasmid. Luciferase activity was measured $30 \mathrm{~h}$ after transfection using a luminometer (Turner Designs).

\section{Reverse Transcription Quantitative Polymerase Chain}

Reaction

Transcription of genes encoding Nqo1 and HO-1 was quantified by reverse transcription quantitative polymerase chain reaction (RT-qPCR). Two days after transfection HEK 293T cells were collected and total RNA was isolated using a PerfectPure RNA Cultured Cell Kit (5Prime). cDNA was synthesized using Mint reverse transcriptase (Evrogen) and random hexamer primers (Evrogen). RT-qPCR was performed on an IQ5 Real-Time PCR Detection System (BioRad) using protocol described previously [21, 28 ]. The primer and probe sequences were as follows: HO-1: 5'-CCAGCAACAAAGTGCAAGATTC-3' (sense primer), $5^{\prime}$-TCACATGGCATAAAGCCCTACAG- ${ }^{\prime}$ (antisense primer), and 5'
(Cy5)-TCTCCGATGGGTCCTTACACTCAGCTTTCT (BHQ2)-3' (probe); Nqo1: 5'-GTCATTCTCTGGCCAATTCAGAGT-3' (sense primer), $5^{\prime}$-TTCCAGGATTTGAATTCGGG-3' (antisense primer), and $5^{\prime}$-(CY5)-ACTGACATATAGCATGGGCACACTCCAGC-(BHQ2)-3' (probe); and $\beta$-actin: $5^{\prime}$-GATCATTGCTCCTCCTGAGC-3' (sense primer), $5^{\prime}$-ACTCCTGCTTGCTGATCCAC-3' (antisense primer), and $5^{\prime}$-(R6G)-CTCGCTGTCCACCTTCCAGCAGAT-(BHQ-1)-3' (probe). A relative quantitative analysis was carried out by comparing the threshold cycle number for target genes and reference $\beta$-actin mRNA, amplified in separate tubes.

\section{Results}

In order to study the effects of NS1 on the redox status of the host cell, a plasmid encoding this protein was constructed. Gene design was performed as previously pro- 


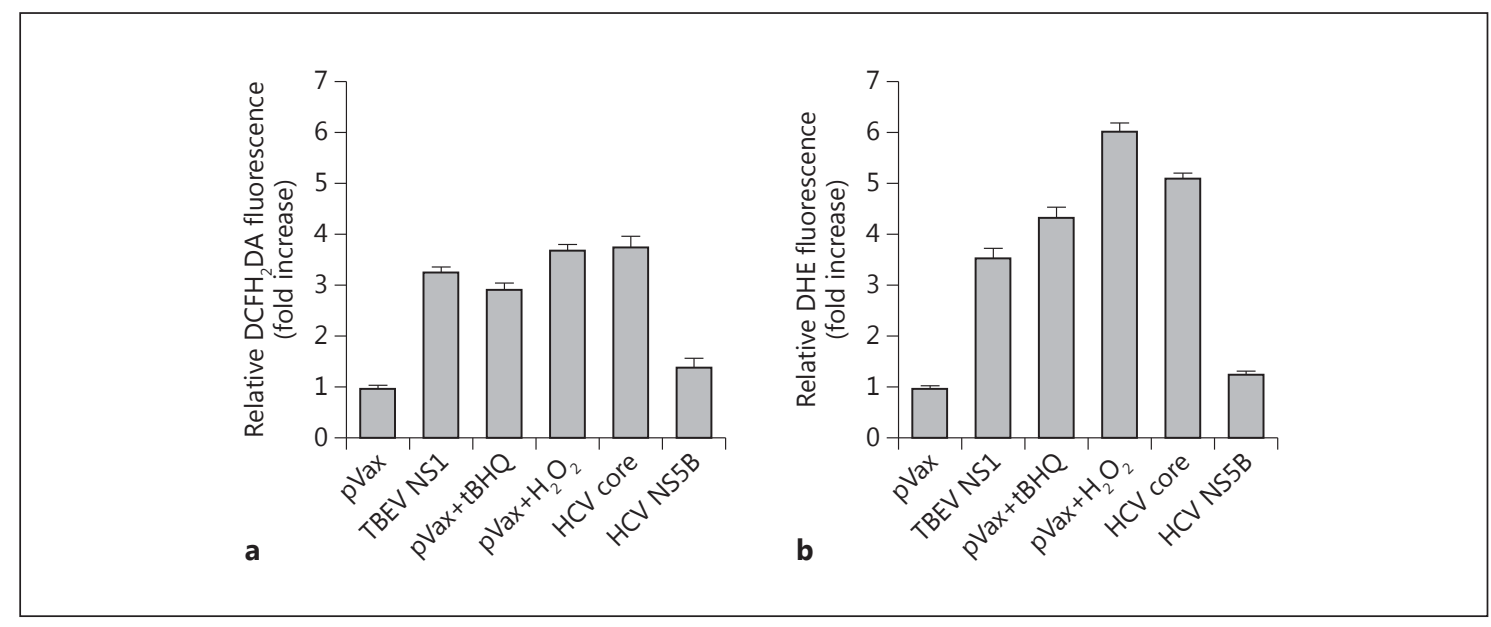

Fig. 2. Expression of nonstructural protein 1 (NS1) induces oxidative stress in cells. HEK $293 \mathrm{~T}$ cells were transfected with pVax or pVax-NS1, and after 2 days ROS were estimated. The production of intracellular ROS in transfected cells was measured by epifluorescence. Two days after transfection the growth medium was removed, and the cells were incubated in Dulbecco's modified Eagle's medium (DMEM) containing $25 \mu \mathrm{M}$ dichlorofluorescein di-

posed by Jacobs et al. [29]. The constructed plasmid encoded a full-length NS1 protein corresponding to the Neudoerfl strain of the virus (GenBank U27495.1) with an ER-translocation sequence on its $\mathrm{N}$-terminus. This gene was synthesized de novo and cloned into a pVax vector. Expression of the NS1 protein was verified by Western blot with monoclonal anti-NS1 antibodies (Fig. 1a). In the lysates of transfected cells, protein of the expected molecular weight (i.e., $47-48 \mathrm{kDa}$ ) was detected. A homodimer of the NS1 protein with an apparent weight of $90-100 \mathrm{kDa}$ was also found in concentrated cell culture fluids as revealed by SDS-PAGE. Intracellular localization studies revealed colocalization of the expressed protein with calreticulin, the marker of ER (Fig. 1b).

HEK 293T cells transfected with the pVax NS1 plasmid were used to study the effect of rotein on ROS production and antioxidant defense. ROS production was assessed by measurement of their levels with oxidation-sensitive dyes on a microplate fluorimeter. For this, $\mathrm{DCFH}_{2} \mathrm{DA}$ and DHE were used. $\mathrm{DCFH}_{2} \mathrm{DA}$ is considered a tool to reveal the general redox status of a cell, whereas DHE is used to quantify the production of the superoxide anion [30]. The control samples were treated with oxidative stress-inducing agents (i.e., tBHQ or hydrogen peroxide). Additional positive and negative controls were obtained by transfecting the cells with the plasmids encoding hepatitis $\mathrm{C}$ virus core and NS5B proteins, respectively, since $\mathrm{HCV}$ core is a acetate $\left(\mathrm{DCFH}_{2} \mathrm{DA}\right)$ (a) or $25 \mu \mathrm{M}$ dihydroethidium (DHE) (b) for $30 \mathrm{~min}$. The fluorescence intensities were measured using a Chameleon $\mathrm{V}$ plate reader. As a positive control the cells were transfected with plasmid expressing $\mathrm{HCV}$ core or with an empty pVax vector followed by treatment with tert-butylhydroquinone ( $\mathrm{tBHQ}$ ). As a negative control the cells were transfected with plasmid expressing HCV NS5B protein.

potent inducer of ROS production whereas NS5B does not affect the redox status of the cell despite its high level of expression [22]. In the case of both dyes, TBEV NS1 triggered a pronounced increase in florescence compared to cells transfected with the empty $\mathrm{pVax}$ vector or expressing the HCV NS5B protein (Fig. 2). The effect was comparable to that of chemical prooxodants. This indicates that NS1 stimulates ROS production in cells.

In order to maintain redox homeostasis, antioxidant mechanisms are often activated in cells during oxidative stress. Enhanced ROS production can activate nuclear factor (erythroid-derived-2)-like 2 (Nrf2), which activates the expression of enzymes implicated in metabolism of low-molecular-weight antioxidants or it can directly scavenge ROS (referred to as phase II enzymes) [31]. It is achieved by binding of Nrf2 to the ARE within the promoters of the respective genes. So, our next step was to explore whether NS1 expression could promote transcription of ARE-dependent genes.

First we used a reporter plasmid encoding a luciferase under an SV40 promoter with a minimum ARE sequence (pP-ARE) [22]. Its cotransfection with the plasmid expressing NS1 strongly stimulated luciferase expression (Fig. 3a). The absence of such stimulation in the case of the $\mathrm{pVax}$ vector or HCV NS5B protein supported the specificity of the effect in the case of TBEV NS1. This indicated activation of ARE-containing promoters in NS1-expressing cells. 


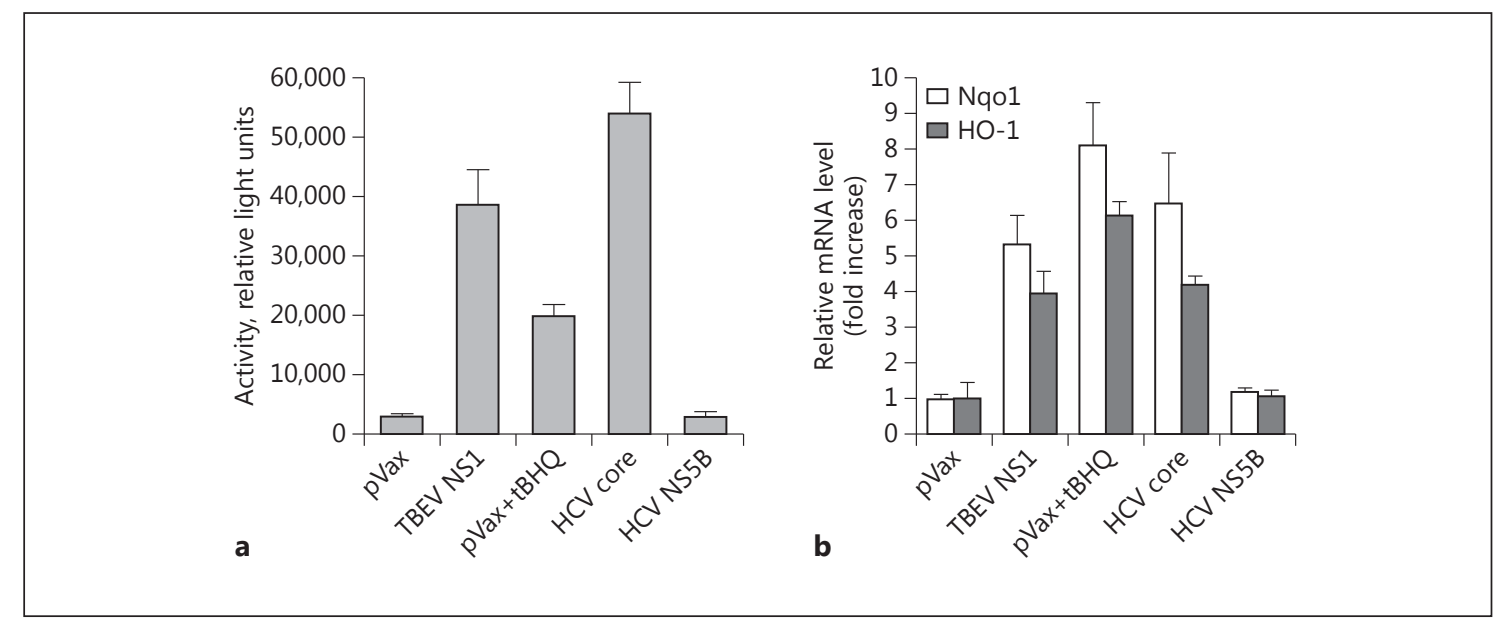

Fig. 3. Nonstructural protein 1 (NS1) activates the nuclear factor (erythroid-derived-2)-like 2/antioxidant response element (Nrf2/ ARE) pathway. a Activation of the ARE-containing promoter in NS1-expressing cells. HEK 293T cells were transfected with a mixture of $\mathrm{pVax}$ or $\mathrm{pVax}-\mathrm{NS} 1$ plasmid and pP-ARE reporter. Thirty hours after transfection the luciferase activity was measured using a luminometer (Turner Designs). b The increase in mRNA levels

Then, the effects of NS1 on the expression of cellular ARE-regulated genes were studied. As such, transcription of genes encoding the phase II detoxifying enzymes NAD $(\mathrm{P})$ $\mathrm{H}$ :quinone oxidoreductase (Nqo1) and heme oxygenase 1 (HO-1) was measured. For this, we transfected HEK 293T cells with pVax or $\mathrm{pVax}$ NS1, and as a positive control the pVax-transfected cells treated with $\mathrm{tBHQ}$ were used. Then relative Nqo1 and HO-1 mRNA levels were measured using quantitative RT-PCR. The gene expression of both enzymes was significantly enhanced in NS1-expressing cells (Fig. 3b). Thus, we showed that NS1 induces oxidative stress and activates the ARE-containing promoter and upregulates the expression of genes of detoxifying enzymes Nqo1 and HO-1.

\section{Discussion}

Virus-host interactions that affect the redox status of a cell can predetermine the fate of the infected cell. High rates of ROS production can cause cell death, whereas maintaining of a proper balance between ROS and oxygen radical scavenging enzymes can promote viral replication as well as survival of the infected cells. The important role of ROS in the pathogenesis of hemorrhage has been shown before for the dengue virus, which, like TBEV, belongs to Flaviviridae family. This virus infection induces apoptosis of phase II detoxifying enzymes Nqo1 and HO-1 in transfected HEK $293 \mathrm{~T}$ cells was measured by RT-qPCR (represented as relative to the expression of $\beta$-actin). As a positive control the cells were transfected with the plasmid expressing HCV core or with an empty $\mathrm{pVax}$ vector followed by treatment with tert-butylhydroquinone (tBHQ). As a negative control the cells were transfected with plasmid expressing HCV NS5B protein.

of endothelial cells though the production of ROS and reactive nitrogen species [32]. Treatment with an inhibitor of NADPH oxidase, one of the ROS-producing enzymes, greatly reduces the severity of the hemorrhage. In addition, the balance between ROS production and activation of an antioxidant defense affects the process of viral replication. Low levels of ROS have been shown to be implicated in mitogenic cell activation during the early phase of lytic and nonlytic virus infection [33]. Studies of dengue virus infection have revealed that the level of oxidative stress and activation of $\mathrm{Nrf} 2$ are critical for controlling the antiviral and apoptotic pathways of infected cells [5]. A reduction of ROS levels using chemical antioxidants in these studies dampened the innate immune responses to dengue virus infection and thus facilitated its replication, whereas the monocyte-derived dendritic cells with chemically increased ROS levels exhibited a lower susceptibility to viral replication. Thus, high ROS levels can be crucial for the pathogenicity of viral infections, and antioxidant treatment can alleviate the damage, though at a possible cost of facilitated viral replication.

Neurons and other cell types of the CNS may be infected by TBEV [2]. Replication of the virus is toxic for the cell but the mechanisms of toxicity are not well defined. In the current study, we have shown that TBEV NS1 protein stimulates ROS production and activates the antioxidant defense Nrf2/ARE pathway in HEK 293T cells. Though 
this cell line was obtained from a human kidney, it was shown to mimic cells of a neuronal lineage phenotype because of its potential to propagate the highly neurotropic viruses, inducible synaptogenesis, functionality of the endogenous neuron-specific voltage-gated channels, and the response to the diverse agonists implicated in neuronal signaling [34]. Mlera et al. [35] used HEK 293T cells to develop a model system for tick-borne flavivirus persistence.

In our experience the ability of the NS1 protein to promote ROS production was comparable to that of the $\mathrm{HCV}$ core protein - a protein that is known to induce pronounced oxidative stress $[7,36]$. This effect is specific and attributed to the intrinsic properties of NS1 and not to its mere overexpression in cells, as shown by the absence of a notable influence of highly expressed HCV NS5B protein on ROS production and of the NRf2/ARE pathway. It is also supported by our previous data showing that $\mathrm{HCV}$ core is expressed at 6- to 7-fold lower levels than NS5B but exhibits strong prooxidant activity [22].

We also evidenced that NS1 activates the Nrf2/ARE pathway in HEK 293T cells. In experiments with a reporter plasmid the effect of NS1 was comparable to that of the HCV core protein and much stronger than that of tBHQ. Again, no ARE activation was detected in $\mathrm{HCV}$ NS5B-cotransfected cells. When measuring the expression of ARE-dependent genes, a 4- to 5-fold upregulation of transcription Nqo1 and HO-1 was seen in NS1-expressing cells. The difference in the effect of NS1 expression and tBHQ in the test with qPCR was less dramatic than in the ARE reporter assay. This could be due to the use of a more simple reporter system as a plasmid with the ARE promoter that perhaps should be used for a semiquantitative assay. However, in our opinion measurement of transcription of genes by RT-qPCR reflects results in a native way due to the natural milieu such as transcriptional factors and self-regulation of the genes.

The mechanisms by which NS1 triggers ROS production are unknown. Since NS1 is an ER-residing transmembrane glycosylated protein [37], it could be speculated that NS1 induces ROS production through ER stress and an unfolded protein response similarly to HCV E1/ E2 glycoproteins or the NS4B transmembrane protein $[38,39]$. However, the main type of ROS in the ER is hydrogen peroxide, whereas our data indicate that NS1 also triggers the production of superoxide anions, as revealed by DHE (Fig. 2b). Therefore, NS1 is likely to affect other ROS-producing systems as well.

Oxidative stress is closely connected to the formation of immunoproteasomes that are required for improved epitope generation for the successive immune clearance of infected cells [40]. In HBV-positive cells a decreased level of the immunoproteasome subunit LMP7 was detected and this effect was Nrf2/ARE mediated [41]. The authors believe that in this case an active antioxidant process in combination with the prevalence of a constitutive proteasome is beneficial for the survival of the infected cell. In our previous studies we have shown that in TBEV NS1expressing cells the concentration of immunoproteasome subunits is increased [42]. This upregulation can be caused by increased ROS as was shown in the current study. An increased immunoproteasome content may improve the viral antigen presentation and make the infected cell more visible for elimination by the immune system. Immunoproteasome activation in combination with oxidative stress induction makes the TBEV-infected cell more susceptible to damage that might accelerate the inflammation process. However, coactivation of ARE-mediated genes by NS1 can balance the redox state in the infected cell and decrease the activation of an antiviral defense.

In the current study, we have shown that expression of the TBEV NS1 protein led to the production of ROS compensated by activation of the antioxidant defense Nrf2/ ARE pathway. The ability to stimulate ROS and an antioxidant response and their proper balance can underlie the different pathogenicity of TBEV strains. A detailed investigation of intracellular proteins and virus-host interactions is important for better understanding TBEV pathophysiology. These data can be useful for further TBE treatment improvement and the development of vaccine candidates against flaviviruses.

\section{Acknowledgements}

This study was supported by the Russian Science Foundation (grant No. 14-50-00060).

\section{Disclosure Statement}

The authors have no conflicts of interest to disclose.

References

1 Kaiser R: Tick-borne encephalitis. Infect Dis Clin North Am 2008;22:561-575.

2 Mandl CW: Steps of the tick-borne encephalitis virus replication cycle that affect neuropathogenesis. Virus Res 2005;111:161-174.

3 Rada B, Leto TL: Oxidative innate immune defenses by Nox/Duox family NADPH oxidases. Contrib Microbiol 2008;15:164-187.

4 Peterhans E: Reactive oxygen species and nitric oxide in viral diseases. Biol Trace Elem Res 1997;56:107-116.
Kuzmenko/Smirnova/Ivanov/

Starodubova/Karpov 
5 Olagnier D, Peri S, Steel C, van Montfoort N, Chiang C, Beljanski V, Slifker M, He Z, Nichols CN, Lin R, Balachandran S, Hiscott J: Cellular oxidative stress response controls the antiviral and apoptotic programs in dengue virus-infected dendritic cells. PLoS Pathog 2014; 10:e1004566.

6 Ibeh BO, Emeka-Nwabunnia IK: Increased oxidative stress condition found in different stages of HIV disease in patients undergoing antiretroviral therapy in Umuahia (Nigeria). Immunopharmacol Immunotoxicol 2012;34: 1060-1066.

7 Ivanov AV, Bartosch B, Smirnova OA, Isaguliants MG, Kochetkov SN: HCV and oxidative stress in the liver. Viruses 2013;5:439-469.

8 Higgs MR, Chouteau P, Lerat H: "Liver let die": oxidative DNA damage and hepatotropic viruses. J Gen Virol 2014;95:991-1004.

9 Gelpi E, Preusser M, Garzuly F, Holzmann H, Heinz FX, Budka H: Visualization of Central European tick-borne encephalitis infection in fatal human cases. J Neuropathol Exp Neurol 2005;64:506-512.

10 Palus M, Formanova P, Salat J, Zampachova E, Elsterova J, Ruzek D: Analysis of serum levels of cytokines, chemokines, growth factors, and monoamine neurotransmitters in patients with tick-borne encephalitis: identification of novel inflammatory markers with implications for pathogenesis. J Med Virol 2015; 87:885-892.

11 Luczaj W, Moniuszko A, Jarocka-Karpowicz I, Pancewicz S, Andrisic L, Zarkovic N, Skrzydlewska E: Tick-borne encephalitis - lipid peroxidation and its consequences. Scand J Clin Lab Invest 2016;76:1-9.

12 Lindenbach BD, Rice CM: Molecular biology of flaviviruses. Adv Virus Res 2003;59:23-61.

13 Pletnev AG, Bray M, Lai CJ: Chimeric tickborne encephalitis and dengue type 4 viruses: effects of mutations on neurovirulence in mice. J Virol 1993;67:4956-4963.

14 Muller DA, Young PR: The flavivirus NS1 protein: molecular and structural biology, immunology, role in pathogenesis and application as a diagnostic biomarker. Antiviral Res 2013;98:192-208.

15 Chung KM, Liszewski MK, Nybakken G, Davis $\mathrm{AE}$, Townsend RR, Fremont $\mathrm{DH}$, Atkinson JP, Diamond MS: West Nile virus nonstructural protein NS1 inhibits complement activation by binding the regulatory protein factor H. Proc Natl Acad Sci USA 2006;103: 19111-19116.

16 Baronti C, Sire J, de Lamballerie X, Querat G: Nonstructural NS1 proteins of several mosquito-borne flavivirus do not inhibit TLR3 signaling. Virology 2010;404:319-330.

17 Vercammen E, Staal J, Beyaert R: Sensing of viral infection and activation of innate immunity by toll-like receptor 3 . Clin Microbial Rev 2008;21:13-25.

18 Wilson JR, de Sessions PF, Leon MA, Scholle F: West Nile virus nonstructural protein 1 inhibits TLR3 signal transduction. J Virol 2008; 82:8262-8271.
19 Turchan-Cholewo J, Dimayuga VM, Gupta S, Gorospe RM, Keller JN, Bruce-Keller AJ: $\mathrm{NADPH}$ oxidase drives cytokine and neurotoxin release from microglia and macrophages in response to HIV-Tat. Antioxid Redox Signal 2009;11:193-204.

20 Deshmane SL, Mukerjee R, Fan S, Del Valle L, Michiels C, Sweet T, Rom I, Khalili K, Rappaport J, Amini S, Sawaya BE: Activation of the oxidative stress pathway by HIV-1 Vpr leads to induction of hypoxia-inducible factor 1alpha expression. J Biol Chem 2009;284: 11364-11373.

21 Isaguliants M, Smirnova O, Ivanov AV, Kilpelainen A, Kuzmenko Y, Petkov S, Latanova A, Krotova O, Engstrom G, Karpov V, Kochetkov S, Wahren B, Starodubova E: Oxidative stress induced by HIV-1 reverse transcriptase modulates the enzyme's performance in gene immunization. Hum Vaccin Immunother 2013;9:2111-2119.

22 Ivanov AV, Smirnova OA, Ivanova ON, Masalova OV, Kochetkov SN, Isaguliants MG: Hepatitis C virus proteins activate NRF2/ARE pathway by distinct ROS-dependent and independent mechanisms in HUH7 cells. PLoS One 2011;6:e24957.

23 Smirnova OA, Ivanova ON, Mukhtarov FS, Tunitskaya VL, Jansons J, Isaguliants MG, Kochetkov SN, Ivanov AV: Analysis of the domains of hepatitis C virus core and NS5A proteins that activate the NRF2/ARE cascade. Acta Naturae 2016;8:155-159.

24 Hristov G, Kramer M, Li J, El-Andaloussi N, Mora R, Daeffler L, Zentgraf H, Rommelaere J, Marchini A: Through its nonstructural protein NS1, parvovirus $\mathrm{H}-1$ induces apoptosis via accumulation of reactive oxygen species. J Virol 2010;84:5909-5922.

25 Tsai CC, Chiu CC, Hsu JD, Hsu HS, Tzang BS, Hsu TC: Human parvovirus B19 NS1 protein aggravates liver injury in NZB/W F1 mice. PLoS One 2013;8:e59724.

26 Alekseeva E, Sominskaya I, Skrastina D, Egorova I, Starodubova E, Kushners E, Mihailova M, Petrakova N, Bruvere R, Kozlovskaya T, Isaguliants M, Pumpens P: Enhancement of the expression of HCV core gene does not enhance core-specific immune response in DNA immunization: advantages of the heterologous DNA prime, protein boost immunization regimen. Genet Vaccines Ther 2009;7:7.

27 Kuzmenko YV, Starodubova ES, Karganova GG, Timofeev AV, Karpov VL: Nonstructural protein 1 of tick-borne encephalitis virus activates the expression of immunoproteasome subunits (in Russian). Mol Biol (Mosk) 2016;50:353-359.

28 Smirnova OA, Ivanova ON, Bartosch B, Valuev-Elliston VT, Mukhtarov F, Kochetkov $\mathrm{SN}$, Ivanov AV: Hepatitis C virus NS5A protein triggers oxidative stress by inducing $\mathrm{NADPH}$ oxidases 1 and 4 and cytochrome P450 2E1. Oxid Med Cell Longev 2016;2016: 8341937.

29 Jacobs SC, Stephenson JR, Wilkinson GW: High-level expression of the tick-borne en- cephalitis virus NS1 protein by using an adenovirus-based vector: protection elicited in a murine model. J Virol 1992;66:2086-2095.

30 Kalyanaraman B, Darley-Usmar V, Davies KJ, Dennery PA, Forman HJ, Grisham MB, Mann GE, Moore K, Roberts LJ 2nd, Ischiropoulos $\mathrm{H}$ : Measuring reactive oxygen and nitrogen species with fluorescent probes: challenges and limitations. Free Radic Biol Med 2012;52:1-6.

31 Ma Q: Role of NRF2 in oxidative stress and toxicity. Annu Rev Pharmacol Toxicol 2013; 53:401-426.

32 Yen YT, Chen HC, Lin YD, Shieh CC, WuHsieh BA: Enhancement by tumor necrosis factor alpha of dengue virus-induced endothelial cell production of reactive nitrogen and oxygen species is key to hemorrhage development. J Virol 2008;82:12312-12324.

33 Peterhans E: Oxidants and antioxidants in viral diseases: disease mechanisms and metabolic regulation. J Nutr 1997;127:962S-965S.

34 Stepanenko AA, Dmitrenko VV: HEK293 in cell biology and cancer research: phenotype, karyotype, tumorigenicity, and stress-induced genome-phenotype evolution. Gene 2015;569:182-190.

35 Mlera L, Offerdahl DK, Martens C, Porcella SF, Melik W, Bloom ME: Development of a model system for tick-borne flavivirus persistence in HEK 293T cells. mBio 2015;6:e00614.

36 Okuda M, Li K, Beard MR, Showalter LA, Scholle F, Lemon SM, Weinman SA: Mitochondrial injury, oxidative stress, and antioxidant gene expression are induced by hepatitis C virus core protein. Gastroenterology 2002; 122:366-375.

37 Akey DL, Brown WC, Jose J, Kuhn RJ, Smith JL: Structure-guided insights on the role of NS1 in flavivirus infection. Bioessays 2015;37: 489-494.

38 Zheng Y, Gao B, Ye L, Kong L, Jing W, Yang $\mathrm{X}, \mathrm{Wu} \mathrm{Z}$, Ye L: Hepatitis C virus non-structural protein NS4B can modulate an unfolded protein response. J Microbiol 2005;43:529536.

39 Chan SW, Egan PA: Hepatitis V virus envelope proteins regulate $\mathrm{CHOP}$ via induction of the unfolded protein response. FASEB J 2005; 19:1510-1512.

40 Kruger E, Kloetzel PM: Immunoproteasomes at the interface of innate and adaptive immune responses: two faces of one enzyme. Curr Opin Immunol 2012;24:77-83.

41 Schaedler S, Krause J, Himmelsbach K, Carvajal-Yepes $M$, Lieder F, Klingel K, Nassal M, Weiss TS, Werner S, Hildt E: Hepatitis B virus induces expression of antioxidant response element-regulated genes by activation of NRF2. J Exp Biol Chem 2010;285:4107441086.

42 Kuzmenko YV, Starodubova ES, Karganova GG, Timofeev AV, Karpov VL: Nonstructural protein 1 of tick-borne encephalitis virus activates the expression of immunoproteasome subunits. Mol Biol 2016;50:307312 . 